\section{È Giuseppe l'eroe di sé stesso}

\author{
La voce dei pazienti
}

AIRP

Qui l'intervista che Max Sisto ha fatto ad Andrea, papà di un piccolo di 9 anni con il Rene Policistico Autosomico Recessivo, rilasciata il 13 ottobre scorso in occasione del viaggio a Venezia con tutti i bambini della Rete del Ma.Re. in età pediatrica. Al link https://www.youtube.com/ watch? $v=k k 8 k s 5 g x D E E \& t=318$ s potrete trovare il video della testimonianza di Andrea.

M.: Andrea, tempo buono insieme quello che stiamo trascorrendo qui in Laguna, tempo buono anche per Giuseppe, per tutti i suoi compagni. Sembra quasi una famiglia allargata: l'AIRP, la Rete del Ma.Re. e il Sogno di Stefano...

A.: Noi siamo una GRANDE FAMIGLIA, così l'abbiamo nominata da quando tre anni fa abbiamo iniziato a fare queste giornate, grazie a Luisa, per incontrarci tutti in Italia, in una città. È stata una richiesta fatta da alcuni genitori proprio per far vedere a questi bambini che non sono soli, che ce ne sono altri come loro, non tanti per fortuna, e noi ci reputiamo una GRANDE FAMIGLIA. Ognuno di noi quando ha un problema o qualcosa che esula da quello che è la vita nostra ordinaria, che già per tutti gli altri non è ordinaria, ci confrontiamo. Grazie ai social adesso abbiamo WhatsApp che ci permette di confrontarci anche con i vari dottori di tutta Italia, per sapere che cosa ne pensano, come si fa con i fratelli, le sorelle, ci facciamo gli auguri di Natale.

M.: Di regola quando c'è un problema grosso di salute le famiglie si rompono, c'è chi non ha il coraggio di andare avanti, si separano mogli e mariti, i figli vanno via. Com'è iniziato invece attraverso la malattia di Giuseppe l'ingresso in questa grande famiglia?

L'ingresso noi lo abbiamo avuto in due steps: perché abbiamo saputo di Giuseppe quando Anna era al settimo mese di gravidanza e siamo stati catapultati nel buio, avevamo 27 e 28 anni. Io e mia moglie non sapevamo proprio niente. Navigando su Internet abbiamo scoperto l'AIRP. All'epoca l'AIRP non era ancora legata al recessivo, ci ha dato delle giuste dritte per poi fare consultazioni genetiche ed essere seguiti al Bambino Gesù di Roma e aiutare Giuseppe. Ci siamo riaffacciati all'AIRP perché appunto avevamo visto tramite Internet e Facebook che si era allargata ai recessivi con la presenza di altri
Giornale di Tecniche Nefrologiche e Dialitiche 2018, Vol. 30(3-4) 22I-222

(C) The Author(s) 2019

Article reuse guidelines:

sagepub.com/journals-permissions

DOI: $10.1177 / 0394936218823527$

journals.sagepub.com/home/gtn

(SSAGE bambini recessivi, ci siamo riaffacciati in maniera, per quanto possiamo attiva, in coincidenza con le problematiche, con le visite e tutto cerchiamo di essere attivi e di aiuto.

M.: Settimo mese di gravidanza vuol dire quasi a 100 metri dal traguardo. Ti ricordi quei momenti uniti poi al momento della nascita e della certezza poi di dover convivere con un problema.

A.: Ricordo tutto. Purtroppo si ricorda tutto da quando l'abbiamo saputo che l'ecografista ci fece una visita al momento a me e a mia moglie per controllare se la nostra situazione renale era a posto, ci diede una prediagnosi stessa al momento dandoci tre malattie di possibilità, di cui una era il recessivo. Ricordo il momento della nascita, mia moglie ha fatto un mese di ricovero in ospedale e Giuseppe quando è nato è andato in terapia intensiva. Non sapevano quando nasceva se viveva o non viveva. Alcuni dottori dicevano che era meglio che morisse altri che poteva nascere con varie malformazioni, momenti duri ... duro è stato confrontarsi con genetisti, confrontarsi con persone che non sapevamo neanche l'esistenza e poi, mano a mano, ti fai le spalle forti e si va avanti e si superano le cose.

M.: Quanti anni ha Giuseppe e come è la sua giornata?

A.: Giuseppe ha 9 anni, è stato inserito il 31 agosto in lista per il trapianto di fegato, stanno valutando se fare anche il trapianto di rene perché questa malattia non è solo renale ma è anche epatica. Questo la rende non maggiore alle altre, perché tutte le malattie sono brutte malattie e ognuno che ha il problema per lui qualsiasi sia l'entità un problema per uno può essere piccolo e per un'altra persona può essere grande; è una montagna che uno tiene davanti $\mathrm{e}$ non sa l'altro quello che ha. Giuseppe la sua giornata è molto faticosa, non riesce a stare al passo con gli altri bambini, anche con gli stessi che hanno la stessa patologia. Il fatto di aver dei valori epatici ed un fegato in sofferenza lo condiziona molto perché il fegato è il laboratorio del corpo, non riesce a produrre delle cose, si sta tirando dietro anche il rene che già va acciaccato anche lui. Quindi è un andare avanti nella caduta e si cerca di ammortizzare in tutti i modi standogli dietro, non si può lasciare a nessuno un bambino del genere, cioè non lo puoi lasciare a una nonna, non lo puoi lasciare a un fratello, non puoi dire 
come gli altri "lasciamo i bambini ai genitori e vado a farmi un weekend con mio marito..." no soprattutto noi che viviamo attaccati al cellulare che puoi essere chiamato in qualsiasi momento per il trapianto. Ma non sono bambini che puoi lasciare ad altri perché è una grande responsabilità.

M.: Abbiamo documentato a luglio scorso al teatro Petruzzelli una grande festa, la festa per i 1500 trapianti di rene in Puglia mentre Papa Francesco era a 50 metri sul lungo mare di Bari ed il mio ricordo di autore di \#Graziedottore è stata la testimonianza di un papà che non conoscevo: "Giorgia nostra figlia, una ragazzina meravigliosa di 14 anni che purtroppo proprio quattro mesi fa come oggi ci ha lasciati per una emorragia celebrale improvvisa. È stata ovviamente una doccia fredda, è stata veramente una tragedia per tutti quanti noi, però quello che ci fa andare avanti ogni giorno con orgoglio è la consapevolezza di aver fatto la scelta giusta, di aver donato gli organi di Giorgia. Giorgia è sempre qui con noi, dentro di noi, Giorgia è in altre persone e questa è la cosa più bella che potevamo fare"

Ora ti chiedo, vista dall'altra parte, dalla parte di colui che sta aspettando per Giuseppe e vista anche da parte di colui che immagino sarà, se non lo è già, un donatore iscritto. Cosa possiamo raccontare a chi dice "eh ti capisco" con una pacca sulla spalla, ma in realtà si limita soltanto a parlare?

A.: In questi anni abbiamo visto tanto, forse troppo; abbiamo visto bambini morire, genitori che hanno perso figli, mogli abbandonate dai mariti, bambini che crescono senza i padri, bambini costretti a stare con il padre perché magari la madre sta a casa con altri figli, dottori che si ritrovano a parlare con genitori che non riescono mentalmente a collegarsi nel recepire determinate informazioni che sono importanti anche se date con il cucchiaino, veramente con il cucchiaino perché ti spiegano dalla $\mathrm{A}$ alla $\mathrm{Z}$ e noi non sappiamo niente. Oggi sai tanto e c'è ancora tanto da imparare e mi rendo conto che le pacche sulle spalle che le persone ti danno, anche quando una persona viene ad esporre il proprio problema, tu non stai in quella persona, non sai il suo stato d'animo, non sai le sue paure, tutto quello che lo coinvolge; quindi la pacca l'accetto perché è un gesto che tutti fanno in senso d'amicizia per dirti praticamente "ok, sei hai bisogno ti aiuto...", poi nella vita impari che tra 100 persone che vengono e nel momento in cui hai bisogno te ne troverai 10 , te ne troverai due, non te ne trovi nessuno. Noi in tutti questi anni abbiamo imparato prima di tutto ad occuparci noi del nostro, guardarci noi il nostro, anche a contatto con i dottori anche a guardare le cose, le valutazioni giornaliere, poi nel caso di necessità si fa uno screening, uno screening per vedere chi effettivamente ti ha dato una pacca sulla spalla e te lo ritrovi e chi praticamente poi lo chiami e non c'è, e allora lo escludi, ma lo escludi non in maniera cattiva, perché tu in quel momento hai bisogno di persone che veramente ti possono aiutare, perché tu hai bisogno di un aiuto concreto e non della pacca in quel momento.

M.: Qual è l'eroe di tuo figlio?

A.: Mio figlio non ha un vero e proprio eroe perché Giuseppe è un bambino molto diverso da tutti gli altri, è un bambino che da piccolo gli psicologi ci hanno detto che già lui pensava al trapianto perché faceva dei disegni di meccanismi, di cambiare pezzi, dobbiamo cambiare questo... un bambino di 3 anni... infatti siamo ancora in contatto con gli psicologi, faceva i disegni per dirci praticamente "guarda questo, collega questo, stacca questo". Ci hanno spiegato che lui è nato praticamente a seguire l'ospedale, a sentire quelle parole un bambino un eroe non glielo trovo perché non vive nel mondo fantastico. Il mondo fantastico è per gli altri bambini. Lui purtroppo vive una dura realtà che alcuni adulti neanche conoscono. Io in prima persona dico che quello che ha passato lui, che passa lui, io non l'ho mai passato. Un bambino di 9 anni che si trova a parlare a scuola con gli altri "mi hanno messo la cannula, qua mi fa male la cannula".

Io dico che è lui il SUO EROE, perché noi glielo diciamo: LUI per noi È IL NOSTRO EROE!

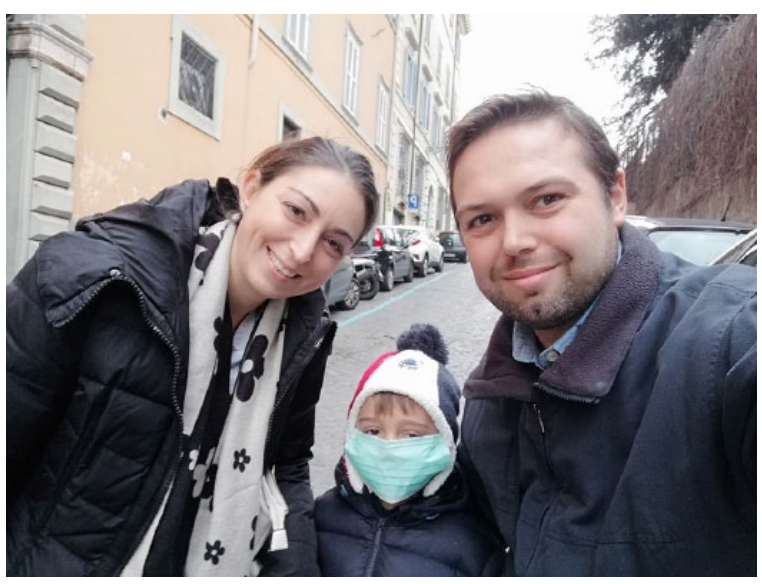

[ndr: Il nostro EROE il 4 novembre ha ricevuto un nuovo fegato. FORZA GIUSEPPE!!!] 